\title{
The Gould-Hopper Polynomials in the Novikov-Veselov equation
}

\author{
Jen-Hsu Chang \\ Department of Computer Science and Information Engineering, \\ National Defense University, \\ Tauyuan, Taiwan
}

April 22, 2022

\begin{abstract}
We use the Gould-Hopper ( $\mathrm{GH}$ ) polynomials to investigate the NovikovVeselov (NV) equation. The root dynamics of the $\sigma$-flow in the NV equation is studied using the GH polynomials and then the Lax pair is found. In particulr, when $N=3,4,5$, one can get the Gold-fish model. The smooth rational solutions of the NV equation are also constructed via the extended Moutard transformation and the GH polynomials. The asymptotic behavior is discussed and then the smooth rational solution of the Liouville equation is obtained.
\end{abstract}

PACS number: 02.30.Ik

Keywords: Gould-Hopper Polynomials, $\sigma$-flows, Lax Equation, Smooth Rational Solutions 


\section{Introduction}

The Novikov-Veselov equation [3, 12, 27, 34] is defined by ( $U$ and $t$ is real)

$$
\begin{aligned}
U_{t} & =\partial_{z}^{3} U+\bar{\partial}_{z}^{3} U-3 \partial_{z}(V U)-3 \bar{\partial}_{z}(\bar{V} U) \\
\bar{\partial}_{z} V & =\partial_{z} U
\end{aligned}
$$

When $z=\bar{z}=x$, we get the famous $\operatorname{KdV}$ equation $(U=V=\bar{V})$

$$
U_{t}=2 U_{x x x}-12 U U_{x}
$$

The equation (1) can be represented as the form of Manakov's triad [23]

$$
H_{t}=[H, A]-B H,
$$

where $H$ is the two-dimensional Schrodinger operator

$$
H=\partial_{z} \bar{\partial}_{z}+U
$$

and

$$
A=\partial_{z}^{3}-V \partial_{z}+\bar{\partial}_{z}^{3}-\bar{V} \bar{\partial}_{z}, \quad B=V_{z}+\bar{V}_{\bar{z}}
$$

It is equivalent to the linear representation

$$
H \psi=0, \quad \partial_{t} \psi=A \psi
$$

We see that the Novikov-Veselov equation (11) preserves a class of the purely potential self-adjoint operators $H$. Here the pure potential means $H$ has no external electric and magnetic fields. The periodic inverse spectral problem for the two-dimensional Schrodinger operator $H$ was investigated in terms of the Riemann surfaces with some group of involutions and the corresponding Prym $\Theta$-functions [6, 14, 19, 15, 26, 31]. On the other hand, it is known that the Novikov-Veselov hierarchy is a special reduction of the two-component BKP hierarchy [28, 33] (and references therein). In [28], the authors showed that the Drinfeld-Sokolov hierarchy of D-type is a reduction of the two-component BKP hierarchy using two different types of pseudo-differential operators, which is different from the Shiota's point of view [31]. Finally, it is worthwhile to notice that the Novikov-Veselov equation (1) is a special reduction of the Davey-Stewartson equation [20, 21].

Let $H \psi=H \omega=0$. Then via the Moutard transformation [1, 24, 25]

$$
\begin{aligned}
U(z, \bar{z}) & \longrightarrow \hat{U}(z, \bar{z})=U(z, \bar{z})+2 \partial \bar{\partial} \ln \left[i \int(\psi \partial \omega-\omega \partial \psi) d z-(\psi \bar{\partial} \omega-\omega \bar{\partial} \psi) d \bar{z}\right] \\
\psi & \longrightarrow \theta=\frac{i}{\omega} \int(\psi \partial \omega-\omega \partial \psi) d z-(\psi \bar{\partial} \omega-\omega \bar{\partial} \psi) d \bar{z}
\end{aligned}
$$


one can construct a new Schrodinger operator $\hat{H}=\partial_{z} \bar{\partial}_{z}+\hat{U}$ and $\hat{H} \frac{1}{\theta}=0$. The extended Moutard transformation was established such that [17, 29]

$$
\begin{aligned}
& \hat{U}(t, z, \bar{z})=U(t, z, \bar{z})+2 \partial \bar{\partial} \ln i W(\psi, \omega), \\
& \hat{V}(t, z, \bar{z})=V(t, z, \bar{z})+2 \partial \partial \ln i W(\psi, \omega)
\end{aligned}
$$

will also satisfy the Novikov-Veselov equation. Here the skew product $W$ is defined by

$$
\begin{aligned}
W(\psi, \omega) & =\int(\psi \partial \omega-\omega \partial \psi) d z-(\psi \bar{\partial} \omega-\omega \bar{\partial} \psi) d \bar{z}+\left[\psi \partial^{3} \omega-\omega \partial^{3} \psi+\omega \bar{\partial}^{3}-\psi \bar{\partial}^{3} \omega\right. \\
& +2\left(\partial^{2} \psi \partial \omega-\partial \psi \partial^{2} \omega\right)-2\left(\bar{\partial}^{2} \psi \bar{\partial} \omega-\bar{\partial} \psi \bar{\partial}^{2} \omega\right)+3 V(\psi \partial \omega-\omega \partial \psi) \\
& -3 \bar{V}(\psi \bar{\partial} \omega-\omega \bar{\partial} \psi)] d t
\end{aligned}
$$

In particular, we can use $U=V=0$ as the seed solution. Then $H=\partial \bar{\partial}$. Let us consider the holomorphic function $P(z, t)$ and satisfy

$$
\frac{\partial P}{\partial t}=\frac{\partial^{3} P}{\partial z^{3}}
$$

Then we have

Theorem 1.1 [32]

Let $\mathcal{P}_{1}(t, z)$ and $\mathcal{P}_{2}(t, z)$ be different holomorphic functions of $z$ and satisfy (4). One defines $\omega_{1}=\mathcal{P}_{1}+\overline{\mathcal{P}}_{1}$ and $\omega_{2}=\mathcal{P}_{2}+\overline{\mathcal{P}}_{2}$. Then

$$
\begin{aligned}
U(t, z, \bar{z}) & =2 \partial \bar{\partial} \ln i W\left(\mathcal{P}_{1}, \mathcal{P}_{2}\right), \\
V(t, z, \bar{z}) & =2 \partial \partial \ln i W\left(\mathcal{P}_{1}, \mathcal{P}_{2}\right)
\end{aligned}
$$

is a solution of Novikov-Veselov equation. Here the skew product $W$ is

$$
\begin{aligned}
W\left(\mathcal{P}_{1}, \mathcal{P}_{2}\right) & =\mathcal{P}_{1} \overline{\mathcal{P}}_{2}-\mathcal{P}_{2} \overline{\mathcal{P}}_{1}+\int\left[\left(\mathcal{P}_{1}^{\prime} \mathcal{P}_{2}-\mathcal{P}_{1} \mathcal{P}_{2}^{\prime}\right) d z+\left(\overline{\mathcal{P}_{1}} \overline{\mathcal{P}}_{2}{ }^{\prime}-\overline{\mathcal{P}}_{1}{ }^{\prime} \overline{\mathcal{P}}_{2}\right) d \bar{z}\right] \\
& +\int\left[\mathcal{P}_{1}^{\prime \prime \prime} \mathcal{P}_{2}-\mathcal{P}_{1} \mathcal{P}_{2}^{\prime \prime \prime}+2\left(\mathcal{P}_{1}^{\prime} \mathcal{P}_{2}^{\prime \prime}-\mathcal{P}_{1}^{\prime \prime} \mathcal{P}_{2}^{\prime}\right)+\overline{\mathcal{P}}_{1} \overline{\mathcal{P}}_{2}^{\prime \prime \prime}-\overline{\mathcal{P}}_{1}^{\prime \prime \prime} \overline{\mathcal{P}}_{2}\right. \\
& \left.+2\left(\overline{\mathcal{P}}_{1}^{\prime \prime} \overline{\mathcal{P}}_{2}{ }^{\prime}-\overline{\mathcal{P}}_{1}^{\prime} \overline{\mathcal{P}}_{2}{ }^{\prime \prime}\right)\right] d t
\end{aligned}
$$

Moreover, if $\mathcal{P}_{1}(t, z)$ and $\mathcal{P}_{2}(t, z)$ are polynomials, then the solution is rational in $z, \bar{z}, t$.

In [2, 7, 8, 13], the rational solutions and line solitons of the Novikov-Veselov equation (1) were constructed by the d-bar dressing method. To get these solutions, the scattering data has to be delta-type and the reality of $U$ also puts some extra constraint on them. In these cases, the $W$-function in (5) and (6) can be expressed 
as a determinant of some matrix.

To study the dispersion relation (4), the authors in [32] introduced the $\sigma$-flows for polynimial-type solutions

$$
\mathbb{P}_{N}(t, z)=z^{N}+\sigma_{1} z^{N-1}+\sigma_{2} z^{N-2}+\cdots+\sigma_{N-1} z+\sigma_{N} .
$$

Then the flow (4) generates a linear flow

$$
\dot{\sigma}_{k}=(N-K+3)(N-k+2)(N-k+1) \sigma_{k-3}, \quad k=1,2,3 \cdots N .
$$

It can be seen that $\sigma_{1}, \sigma_{2}$ are conserved quantities. Indeed, $\sigma_{1}, \sigma_{2}, \cdots, \sigma_{N}$ are the elementary symmetric polynomials in the roots $q_{1}, q_{2}, \cdots, q_{N}$ of $\mathbb{P}_{N}(z)$ :

$$
\begin{aligned}
& \sigma_{1}(\vec{q})=-\sum_{i=1}^{N} q_{i}, \quad \sigma_{2}(\vec{q})=\sum_{i<j} q_{i} q_{j}, \\
& \sigma_{3}(\vec{q})=-\sum_{i<j<k} q_{i} q_{j} q_{k}, \cdots, \sigma_{N}(\vec{q})=(-1)^{N} q_{1} q_{2} \cdots q_{N} .
\end{aligned}
$$

The integrable (even linear) evolution of $\vec{\sigma}=\left(\sigma_{1}, \sigma_{2}, \cdots, \sigma_{N}\right)$ induces a dynamical system on the symmetric product $S^{N} C$ of the complex roots . We call such a dynamical system on $S^{N} C$ a $\sigma$-system.

From (7), we see that given two solutions $\mathcal{P}_{1}(t, z)$ and $\mathcal{P}_{2}(t, z)$, one obtains a solution of the Novikov-Veselov solution by a substitution of $e^{i \lambda_{1}} \mathcal{P}_{1}(t, z)$ and $e^{i \lambda_{2}} \mathcal{P}_{2}(t, z)$ into (7). The $\lambda_{1}$ and $\lambda_{2}$ defined here are real-valued constants. Therefore, to each pair of holomorphic solutions of (4), we can get an $\left(S^{1} \times S^{1}\right)$-family of solutions to the Novikiv-Veselov equation [32].

The paper is organized as follows. In section 2, we describe the Gould-Hopper polynomials using the generating function and establish the recursive relation. In section 3, one studies the root dynamics of $\sigma$-flow and the Lax pair is constructed. Also, the asymptotic behavior is discussed. In section 4, the smooth rational solutions are found using the Gould-Hopper polynomials and their asymptotic behavior is investigated. Section 5 is devoted to the concluding remarks.

\section{Gould-Hopper Polynomials}

In this section, we introduce the Gould-Hopper polynomials and use them to get the solutions of (4). To investigate the polynomial-type solutions of (4), inspired by the work in [11], one utilizes the Gould-Hopper polynomials [10]. The generating function of the Gould-Hopper polynomials $P_{N}(t, z)$ is

$$
e^{\lambda z+\lambda^{3} t}=\sum_{N=0}^{\infty} P_{N}(t, z) \frac{\lambda^{N}}{N !} .
$$


Indeed, the Gould-Hopper polynomials $P_{N}(t, z)$ has the operator representation

$$
P_{N}(t, z)=e^{t \partial_{z}^{3}} z^{N}=\left[1+t \partial_{z}^{3}+\frac{t^{2} \partial_{z}^{6}}{2 !}+\frac{t^{3} \partial_{z}^{9}}{3 !}+\frac{t^{4} \partial_{z}^{12}}{4 !}+\cdots\right] z^{N}
$$

Then one gets

$$
e^{t \partial_{z}^{3}}\left(e^{\lambda z}\right)=e^{\lambda z+\lambda^{3} t}
$$

We remark that in general the Gould-Hopper polynomials are defined by $P_{N}^{(m)}(t, z)=$ $e^{t \partial_{z}^{m}} z^{N}$. Here we take $m=3$.

One notices that the Gould-Hopper polynomials $P_{N}(t, z)$ are characterized by (41) and $P_{N}(0, z)=z^{N}$. For example,

$$
\begin{aligned}
P_{0} & =1, \quad P_{1}=z, \quad P_{2}=z^{2}, \quad P_{3}=z^{3}+6 t, \quad P_{4}=z^{4}+24 t z \\
P_{5} & =z^{5}+60 t z^{2}, \quad P_{6}=z^{6}+120 t z^{3}+360 t^{2} \\
P_{7} & =z^{7}+210 t z^{4}+2520 z, \quad P_{8}=z^{8}+336 t z^{5}+10080 t^{2} z^{2} \\
P_{9} & =z^{9}+504 t z^{6}+30240 t^{2} z^{3}+60480 t^{3} \\
P_{10} & =z^{10}+720 t z^{7}+75600 t^{2} z^{4}+604800 t^{3} z
\end{aligned}
$$

Actually, we have

$$
\begin{aligned}
P_{N}(t, z) & =N ! \sum_{k=0}^{[N / 3]} \frac{t^{k} z^{N-3 k}}{k !(N-3 k) !} \\
\frac{d P_{N}(t, z)}{d z} & =N P_{N-1}(t, z)
\end{aligned}
$$

From the operation calculus, one has

$$
\left(z+3 t \partial_{z}^{2}\right) P_{N-1}(t, z)=P_{N}(t, z), \quad N \geq 1
$$

Hence we yield the recursive relation

$$
P_{N}(t, z)=z P_{N-1}(t, z)+3 t(N-1)(N-2) P_{N-3}(t, z) .
$$

We can see that if we consider the equation (4) with the initial data of analytical function

$$
P(0, z)=\sum_{N=0}^{\infty} \alpha_{N} z^{N}
$$

then the formal solution is

$$
P(t, z)=e^{t \partial_{z}^{3}} \sum_{N=0}^{\infty} \alpha_{N} z^{N}=\sum_{N=0}^{\infty} \alpha_{N} P_{N}(t, z) .
$$


The successive operation of the operator $\left(z+3 t \partial_{z}^{2}\right)$ on the solution (15) can help us construct more solutions of (44). For example, if $P(0, z)=\sin z$, then we have,

$$
\begin{aligned}
e^{t \partial_{z}^{3}} \sin z & =e^{t \partial_{z}^{3}} \sum_{N=0}^{\infty} \frac{(-1)^{N}}{(2 N+1) !} z^{2 N+1}=\sum_{N=0}^{\infty} \frac{(-1)^{N}}{(2 N+1) !} P_{2 N+1}(t, z) \\
& =\sin (z-t) .
\end{aligned}
$$

The last equation uses (11). Hence

$$
\left(z+3 t \partial_{z}^{2}\right)^{N} \sin (z-t), \quad N=0,1,2,3,4, \cdots
$$

are also solutions of (4).

Remark: Let's define

$$
\varphi(\lambda)=e^{\lambda z+\lambda^{3} t}-e^{-\lambda z-\lambda^{3} t}=2 \sinh \left(\lambda z+\lambda^{3} t\right) .
$$

Then $\varphi(\lambda)$ satisfies

$$
\varphi(\lambda)_{z z}=\lambda^{2} \varphi(\lambda), \quad \varphi(\lambda)_{t}=\varphi(\lambda)_{z z z} .
$$

On expanding

$$
\varphi(\lambda)=\sum_{i=0}^{\infty} \phi_{i} \lambda^{2 i+1}
$$

one has

$$
\phi_{0, z z}=0, \quad \phi_{i+1, z z}=\phi_{i}, \quad \phi_{i, t}=\phi_{i, z z z}, \quad i \geq 0 .
$$

Actually,

$$
\phi_{i}=\sum_{k=0}^{\left[\frac{2 i+1}{3}\right]} \frac{1}{k !(2 i+1-3 k) !} z^{2 i+1-3 k} t^{k}, \quad i \geq 0 .
$$

It is known that $\phi_{i}$ can be used to construct the Wronskian solutions of the KdV equation. The details can be found in [22].

\section{Root Dynamics of $\sigma$-flows}

In this section, one uses the Gould-Hopper polynomials to study the root dynamics of the $\sigma$-flows (91).

Let's write (8) as

$$
\mathbb{P}_{N}(t, z)=\left(z-q_{1}(t)\right)\left(z-q_{2}(t)\right) \cdots\left(z-q_{N}(t)\right) .
$$

Then from the equation (41), one gets the root dynamics

$$
\dot{q}_{j}=-6 \sum_{m<n, \quad j_{j \neq m, n}}^{N} \frac{1}{\left(q_{j}-q_{m}\right)\left(q_{j}-q_{n}\right)}
$$


For example, when $\mathrm{N}=3$, we have

$$
\begin{aligned}
& \dot{q}_{1}=-6 \frac{1}{\left(q_{1}-q_{2}\right)\left(q_{1}-q_{3}\right)} \\
& \dot{q}_{2}=-6 \frac{1}{\left(q_{2}-q_{1}\right)\left(q_{2}-q_{3}\right)} \\
& \dot{q_{3}}=-6 \frac{1}{\left(q_{3}-q_{1}\right)\left(q_{3}-q_{2}\right)}
\end{aligned}
$$

For $\mathrm{N}=4$, we get

$$
\begin{aligned}
& \dot{q}_{1}=-6\left[\frac{1}{\left(q_{1}-q_{2}\right)\left(q_{1}-q_{3}\right)}+\frac{1}{\left(q_{1}-q_{3}\right)\left(q_{1}-q_{4}\right)}+\frac{1}{\left(q_{1}-q_{2}\right)\left(q_{1}-q_{4}\right)}\right] \\
& \dot{q_{2}}=-6\left[\frac{1}{\left(q_{2}-q_{1}\right)\left(q_{2}-q_{3}\right)}+\frac{1}{\left(q_{2}-q_{3}\right)\left(q_{2}-q_{4}\right)}+\frac{1}{\left(q_{2}-q_{1}\right)\left(q_{2}-q_{4}\right)}\right] \\
& \dot{q_{3}}=-6\left[\frac{1}{\left(q_{3}-q_{1}\right)\left(q_{3}-q_{2}\right)}+\frac{1}{\left(q_{3}-q_{1}\right)\left(q_{3}-q_{4}\right)}+\frac{1}{\left(q_{3}-q_{2}\right)\left(q_{3}-q_{4}\right)}\right] \\
& \dot{q_{4}}=-6\left[\frac{1}{\left(q_{4}-q_{2}\right)\left(q_{4}-q_{3}\right)}+\frac{1}{\left(q_{4}-q_{1}\right)\left(q_{4}-q_{2}\right)}+\frac{1}{\left(q_{4}-q_{1}\right)\left(q_{1}-q_{3}\right)}\right]
\end{aligned}
$$

We notice that since $\sigma_{1}$ and $\sigma_{2}$ are conserved quantities, one knows that

$$
\sum_{i=1}^{N} q_{i}, \quad \sum_{i=1}^{N} q_{i}^{2}
$$

are conserved densities of (16).

Now, we can investigate the properties of the root dynamics (16) by the GouldHopper polynomials:

- Initial Value Problem : The root dynamics of $\sigma$-flow can be solved by

$$
\begin{aligned}
\mathbb{P}_{N}(t, z) & =\left(z-q_{1}(t)\right)\left(z-q_{2}(t)\right) \cdots\left(z-q_{N}(t)\right) \\
& =P_{N}(t, z)+C_{1} P_{N-1}(t, z)+\cdots+C_{N} P_{0}(t, z),
\end{aligned}
$$

where the constants $C_{1}, C_{2}, \cdots, C_{N-1}, C_{N}$ are determined by the initial values of $q_{1}(0), q_{2}(0), \cdots, q_{N}(0)$, that is,

$$
\begin{aligned}
C_{1} & =-\sum_{i=1}^{N} q_{i}(0), \quad C_{2}=\sum_{i<j} q_{i}(0) q_{j}(0), \\
C_{3} & =-\sum_{i<j<k} q_{i}(0) q_{j}(0) q_{k}(0), \quad \cdots, \\
C_{N} & =(-1)^{N} q_{1}(0) q_{2}(0) \cdots q_{N}(0) .
\end{aligned}
$$

Therefore, it is seen that the solutions $q_{1}(t), q_{2}(t), \cdots, q_{N}(t)$ can be obtained algebraically. 
- Lax pair:

Firstly, we study the root dynamics of the Gould-Hopper polynomials, which correspond to the initial values $q_{1}(0)=q_{2}(0)=\cdots=q_{N}(0)=0$.

Let's define the $N \times N$ matrix by

$$
X(t)= \begin{cases}a_{i, i+1}=1, & \text { if } i=1,2,3, \cdots, N \\ a_{i, i-2}=-3 t(i-1)(i-2), & \text { if } i=3,4 \cdots, N-1 \\ 0, & \text { otherwise }\end{cases}
$$

Then from the recursive relation (14), one knows that

$$
P_{N}(t, z)=\operatorname{det}\left(z I_{N}-X(t)\right) .
$$

For example, when $N=3$,

$$
X(t)=\left(\begin{array}{ccc}
0 & 1 & 0 \\
0 & 0 & 1 \\
-6 t & 0 & 0
\end{array}\right)
$$

$\mathrm{N}=4$,

$$
X(t)=\left(\begin{array}{cccc}
0 & 1 & 0 & 0 \\
0 & 0 & 1 & 0 \\
-6 t & 0 & 0 & 1 \\
0 & -18 t & 0 & 0
\end{array}\right)
$$

$\mathrm{N}=5$,

$$
X(t)=\left(\begin{array}{ccccc}
0 & 1 & 0 & 0 & 0 \\
0 & 0 & 1 & 0 & 0 \\
-6 t & 0 & 0 & 1 & 0 \\
0 & -18 t & 0 & 0 & 1 \\
0 & 0 & -36 t & 0 & 0
\end{array}\right)
$$

We can write $X(t)$ as

$$
X(t)=R(t) Q R^{-1}(t),
$$

where $Q=\operatorname{diag}\left(q_{1}(t), q_{2}(t), \cdots, q_{N}(t)\right.$ and

$$
R(t)=\left(\begin{array}{ccccc}
P_{0}\left(q_{1}, t\right) & P_{0}\left(q_{2}, t\right) & P_{0}\left(q_{3}, t\right) & \cdots & P_{0}\left(q_{N}, t\right) \\
P_{1}\left(q_{1}, t\right) & P_{1}\left(q_{2}, t\right) & P_{1}\left(q_{3}, t\right) & \cdots & P_{1}\left(q_{N}, t\right) \\
P_{2}\left(q_{1}, t\right) & P_{2}\left(q_{2}, t\right) & P_{2}\left(q_{3}, t\right) & \cdots & P_{2}\left(q_{N}, t\right) \\
\vdots & & & & \\
P_{N}\left(q_{1}, t\right) & P_{N}\left(q_{2}, t\right) & P_{N}\left(q_{3}, t\right) & \cdots & P_{N}\left(q_{N}, t\right)
\end{array}\right) .
$$


For instance, when $N=3$,

$$
R(t)=\left(\begin{array}{ccc}
1 & 1 & 1 \\
q_{1} & q_{2} & q_{3} \\
q_{1}^{2} & q_{2}^{2} & q_{3}^{2}
\end{array}\right)
$$

and $\mathrm{N}=4$,

$$
R(t)=\left(\begin{array}{cccc}
1 & 1 & 1 & 1 \\
q_{1} & q_{2} & q_{3} & q_{4} \\
q_{1}^{2} & q_{2}^{2} & q_{3}^{2} & q_{4}^{2} \\
q_{1}^{3}+6 t & q_{2}^{3}+6 t & q_{3}^{3}+6 t & q_{4}^{3}+6 t
\end{array}\right)
$$

$\mathrm{N}=5$,

$$
R(t)=\left(\begin{array}{ccccc}
1 & 1 & 1 & 1 & 1 \\
q_{1} & q_{2} & q_{3} & q_{4} & q_{5} \\
q_{1}^{2} & q_{2}^{2} & q_{3}^{2} & q_{4}^{2} & q_{5}^{2} \\
q_{1}^{3}+6 t & q_{2}^{3}+6 t & q_{3}^{3}+6 t & q_{4}^{3}+6 t & q_{5}^{3}+6 t \\
q_{1}^{4}+24 t & q_{2}^{4}+24 t & q_{3}^{4}+24 t & q_{4}^{4}+24 t & q_{5}^{4}+24 t
\end{array}\right)
$$

From the initial value problem (17), we notice that the polynomials $t^{n}$ can be replaced by the elementary symmetric polynomials of the roots $q_{1}, q_{2}, \cdots, q_{N}$. Hence one has $R(\vec{q})$. It can be seen that

$$
\dot{X}(t)=R L R^{-1},
$$

where

$$
L=\dot{Q}+[M, Q], \quad M=R^{-1} \dot{R} .
$$

For example, when $\mathrm{N}=3$,

$$
L(t)=\left(\begin{array}{ccc}
\dot{q}_{1} & \dot{q}_{2} \frac{q_{2}-q_{3}}{q_{3}-q_{1}} & \dot{q_{3}} \frac{q_{3}-q_{2}}{q_{2}-q_{1}} \\
\dot{q}_{1} \frac{q_{1}-q_{3}}{q_{3}-q_{2}} & \dot{q}_{2} & \dot{q}_{3} \frac{q_{3}-q_{1}}{q_{1}-q_{2}} \\
\dot{q}_{1} \frac{q_{1}-q_{2}}{q_{2}-q_{3}} & \dot{q}_{2} \frac{q_{2}-q_{1}}{q_{1}-q_{3}} & \dot{q}_{3}
\end{array}\right) ;
$$

and $\mathrm{N}=4$, using the Maple software,

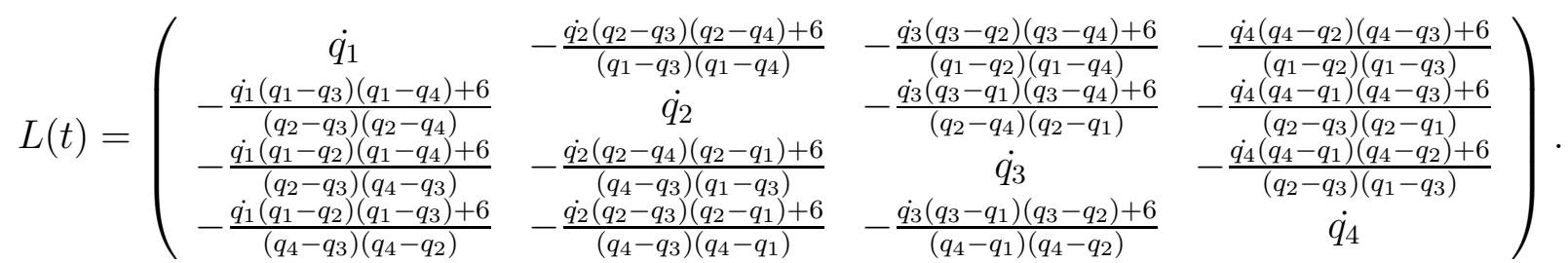

Since

$$
\dot{X}(t)=\frac{d X(t)}{d t}= \begin{cases}a_{i, i-2}=-3(i-1)(i-2), & \text { if } i=3,4 \cdots, N-1 ; \\ 0, & \text { otherwise, }\end{cases}
$$


we know $\dot{X}(t)$ is a nilpotent matrix and hence $L$ is a nilpotent one, too. So

$$
\operatorname{tr}\left(L^{r}\right)=\operatorname{tr}[\dot{X}(t)]^{r}=0, \quad r=1,2,3, \ldots, . .
$$

Actually, a simple calculation yields

$$
L^{\left[\frac{N}{2}\right]+1}=0, \quad N \geq 3,
$$

where $[\alpha]$ means the integer part of $\alpha$. Now,

$$
\frac{d^{2} X(t)}{d t^{2}}=0
$$

will imply the Lax equation

$$
\frac{d L(t)}{d t}=[L, M]
$$

For $N=3,4,5$, we see that, by the Maple software, $q_{i}$ satisfies the following Goldfish model [5], a limiting case of the Ruijesenaars-Schneider system:

$$
\ddot{q}_{i}=2 \sum_{j \neq i} \frac{q_{i} q_{j}}{q_{i}-q_{j}} .
$$

The reason is that $P_{i}, i=3,4,5$ are linear in $t$-variable(see the appendix). For $N=6$, we have from the diagonal terms of the Lax equation (20)

$$
\ddot{q}_{i}=2 \sum_{j \neq i}^{6} \frac{q_{i} q_{j}}{q_{i}-q_{j}}+\frac{\sum_{j=1}^{6}(\text { some quadratic terms of } \vec{q}) q_{j}+720}{\prod_{i \neq j}^{6}\left(q_{i}-q_{j}\right)} .
$$

Secondly, we consider the general case. Let's define the 2D Appell polynomials $\mathbb{G}_{n}(z, t)$ by means of the generating function [4]:

$$
A(\lambda) e^{\lambda z+\lambda^{3} t}=\sum_{n=0}^{\infty} \mathbb{G}_{n}(z, t) \frac{\lambda^{n}}{n !},
$$

where

$$
A(\lambda)=\sum_{0}^{N} \frac{\Gamma_{k}}{k !} \lambda^{k}
$$

$\Gamma_{k}^{\prime} s$ being constants and $\boldsymbol{\Gamma}_{0}=1$. Then one has the following formula, noting that $\left(\begin{array}{l}N \\ h\end{array}\right)=\left(\begin{array}{c}N \\ N-h\end{array}\right)$,

$$
\mathbb{G}_{N}=\sum_{h=0}^{N}\left(\begin{array}{c}
N \\
N-h
\end{array}\right) \boldsymbol{\Gamma}_{N-h} P_{h}(z, t)
$$


It's easy to see that the polynomials $\mathbb{G}_{n}(z, t)$ also satisfy the linear equation (44). When comparing (17) with (23), we have

$$
\Gamma_{N-h}=\frac{C_{N-h}}{\left(\begin{array}{c}
N \\
N-h
\end{array}\right)} .
$$

Now, it's suitable to introduce the coefficients of the Taylor expansion

$$
\frac{A^{\prime}(\lambda)}{A(\lambda)}=\sum_{n=0}^{\infty} \alpha_{n} \frac{\lambda^{n}}{n !} .
$$

It can be seen that the coefficients $\alpha_{n}$ can be expressed by $\boldsymbol{\Gamma}_{0}, \boldsymbol{\Gamma}_{1}, \cdots, \boldsymbol{\Gamma}_{n+1}$ (or the initial values (24) ). For examples,

$$
\begin{aligned}
& \alpha_{0}=\boldsymbol{\Gamma}_{1}, \quad \alpha_{1}=\boldsymbol{\Gamma}_{2}-\boldsymbol{\Gamma}_{1}^{2}, \quad \alpha_{2}=\boldsymbol{\Gamma}_{3}-3 \boldsymbol{\Gamma}_{1} \boldsymbol{\Gamma}_{2}+2 \boldsymbol{\Gamma}_{1}^{3}, \\
& \alpha_{3}=\boldsymbol{\Gamma}_{4}+12 \boldsymbol{\Gamma}_{1}^{2} \boldsymbol{\Gamma}_{2}-4 \boldsymbol{\Gamma}_{1} \boldsymbol{\Gamma}_{3}-3 \boldsymbol{\Gamma}_{2}^{2}-6 \boldsymbol{\Gamma}_{1}^{4},
\end{aligned}
$$

The recurrence relation for the $2 \mathrm{D}$ Appell polynomial $\mathbb{G}_{N}(z, t)$ can be written as follows [4]:

$$
\begin{aligned}
\mathbb{G}_{0}(z, t) & =1 \\
\mathbb{G}_{N}(z, t) & =\left(z+\alpha_{0}\right) \mathbb{G}_{N-1}(z, t)+3 t(N-1)(N-2) \mathbb{G}_{N-3}(z, t) \\
& +\sum_{k=0}^{N-2}\left(\begin{array}{c}
N-1 \\
k
\end{array}\right) \alpha_{N-k-1} \mathbb{G}_{k}(z, t) .
\end{aligned}
$$

A simple calculation can yield

$$
\begin{aligned}
\mathbb{G}_{0}(z, t) & =1, \quad \mathbb{G}_{1}(z, t)=z+\alpha_{0}, \quad \mathbb{G}_{2}(z, t)=\left(z+\alpha_{0}\right)^{2}+\alpha_{1} \\
\mathbb{G}_{3}(z, t) & =\left(z+\alpha_{0}\right)^{3}+3 \alpha_{1}\left(z+\alpha_{0}\right)+\alpha_{2}+6 t \\
\mathbb{G}_{4}(z, t) & =\left(z+\alpha_{0}\right)^{4}+6 \alpha_{1}\left(z+\alpha_{0}\right)^{2}+\left(4 \alpha_{2}+24 t\right)\left(z+\alpha_{0}\right)+\alpha_{3}+3 \alpha_{1}^{2} \\
\mathbb{G}_{5}(z, t) & =\left(z+\alpha_{0}\right)^{5}+10 \alpha_{1}\left(z+\alpha_{0}\right)^{3}+\left(10 \alpha_{2}+60 t\right)\left(z+\alpha_{0}\right)^{2} \\
& +\left(5 \alpha_{3}+15 \alpha_{1}^{2}\right)\left(z+\alpha_{0}\right)+60 t \alpha_{1}+10 \alpha_{1} \alpha_{2}+\alpha_{4} .
\end{aligned}
$$

When $A(\lambda)=1$, this recursive relation becomes (14). Hence the relation (25) is a generalization of (14) for arbitrary initial data. The matrix corresponding 
to (18) can be constructed as follows:

$$
X(t)= \begin{cases}a_{i, i+1}=1, & \text { if } i=1,2,3, \cdots, N-1 ; \\
a_{i, i}=-\alpha_{0}, & \text { if } i=1,2,3, \cdots, N ; \\
a_{i, i-1}=-\left(\begin{array}{c}
i-1 \\
i-2
\end{array}\right) \alpha_{1}, & \text { if } i=2,3,4, \cdots, N ; \\
a_{i, i-2}=-\left(\begin{array}{c}
i-1 \\
i-3
\end{array}\right)\left(6 t+\alpha_{2}\right), & \text { if } i=3,4,5, \cdots, N ; \\
a_{i, i-3}=-\left(\begin{array}{c}
i-1 \\
i-4
\end{array}\right) \alpha_{3}, & \text { if } i=4,5,6, \cdots, N ; \\
\vdots & \\
a_{i, i-k}=-\left(\begin{array}{c}
i-1 \\
i-k-1
\end{array}\right) \alpha_{k}, & \text { if } i=k+1, k+2, k+3, \cdots, N ; \\
\vdots & \\
a_{N, 1}=-\alpha_{N-1}\end{cases}
$$

Similarly, one has

$$
\mathbb{G}_{N}(z, t)=\operatorname{det}\left(z I_{N}-X(t)\right) .
$$

For instance, when $N=5$, we get

$$
X(t)=\left(\begin{array}{ccccc}
-\alpha_{0} & 1 & 0 & 0 & 0 \\
-\alpha_{1} & -\alpha_{0} & 1 & 0 & 0 \\
-\left(6 t+\alpha_{2}\right) & -2 \alpha_{1} & -\alpha_{0} & 1 & 0 \\
-\alpha_{3} & -\left(18 t+3 \alpha_{2}\right) & -3 \alpha_{1} & -\alpha_{0} & 1 \\
-\alpha_{4} & -4 \alpha_{3} & -\left(36 t+6 \alpha_{2}\right) & -4 \alpha_{1} & -\alpha_{0}
\end{array}\right) .
$$

Also, one can write $X(t)$ as

$$
X(t)=R(t) Q R^{-1}(t),
$$

where $Q=\operatorname{diag}\left(q_{1}(t), q_{2}(t), \cdots, q_{N}(t)\right.$ and $R(t)$ is defined as (19) with $P_{m}\left(q_{i}, t\right)$ being replaced by $\mathbb{G}_{m}\left(q_{i}, t\right)$. Then one follows the previous procedures and finally can get the Lax equation (20) for general case. Therefore the root dynamics (16) is Lax-integrable. But the computations are more involved and one doesn't pursuit them here.

We notice here that for $N=3,4,5$ the root dynamics of $\mathbb{G}_{N}$ also satisfies the Gold-fish model (21).

- Asymptotic behavior

It is known that the Gould-Hopper polynomial $P_{N}(t . z)$ has the scaling property:

$$
P_{N}(t, z)=t^{\frac{N}{3}} \hat{P}_{N}\left(\frac{z}{t^{1 / 3}}\right)
$$

where $\hat{P}_{N}(\eta)$ is the so-called Appell polynomials [4] (and references therein) in $\eta=\frac{z}{t^{1 / 3}}$. For example,

$$
\begin{aligned}
P_{8}(t, z) & =z^{8}+336 t z^{5}+10080 t^{2} z^{2}=t^{\frac{8}{3}}\left[\eta^{8}+336 \eta^{5}+10080 \eta^{2}\right] \\
& =t^{\frac{8}{3}} \hat{P}_{8}(\eta)
\end{aligned}
$$


Then the k-th zero $\lambda_{N}^{(k)}$ of $\hat{P}_{N}(\eta)$ determines the dynamics of the root $q_{k}$, i.e.,

$$
q_{k}(t)=\lambda_{N}^{(k)} t^{1 / 3}
$$

Since $\hat{P}_{N}\left(\xi \lambda_{N}^{(k)}\right)=0, \xi^{3}=1$, one knows that the roots $q_{k}$ are located on the circles in the plane with time dependent radius or fixed at the origin. Finally, from the Initial value Problem (17) and (26) , we know that when $t \rightarrow \infty$ and $z \rightarrow \infty$ such that $|z|^{3} / t \rightarrow$ constant, $P_{N}(t, z)$ plays the dominant role. Then one yields

$$
q_{k}(t) \rightarrow \lambda_{N}^{(k)} t^{1 / 3}
$$

Consequently, the roots asymptotically will follow diagonal lines.

\section{Smooth Rational Solutions of Novikov-Vaselov equation}

In [32, the blow-up solution of the Novikov-Vaselov equation via the extended Moutard transformation (7) is constructed. In this section, we establish smooth rational solutions for all time by the Gould -Hopper polynomials (12). Some calculations below need the Maple software.

- Example 1

Let $\mathcal{P}_{1}=z^{2}+z+1$ and $\mathcal{P}_{2}=-i z^{2}-2 i z$. Then a simple calculation can yield the imaginary part of $W$ in (7)

$$
M(x, y, t)=\left(x^{2}+y^{2}\right)^{2}+\frac{8}{3} x^{3}+4 x y^{2}+4 x^{2}+4 x+4 t+100
$$

From (27), we can see that

$$
M(x, y, 0) \approx\left(x^{2}+y^{2}\right)^{2} \quad \text { near } \quad r=\sqrt{x^{2}+y^{2}}=\infty .
$$

It can be verified that $M(x, y, 0)$ is positive for all $\mathbb{R}^{2}$. Also, $M(x, y, t)$ is positive for all $\mathbb{R}^{2}$ at any fixed time $t \geq 0$. Then the solution $U$ in (5) of the Novikov-Veselov equation (10) is

$$
U=\frac{M_{1}}{M_{2}}
$$

where

$$
\begin{aligned}
M_{1} & =-12\left(294+600 x^{2}+588 y^{2}+8 x^{3}+888 x+12 t+3 x^{4}-6 x^{2} y^{2}-2 x^{3} y^{2}\right. \\
& \left.+24 x^{2} t-3 y^{4} x+24 y^{2} t+36 x t-3 y^{4}+x^{5}\right)
\end{aligned}
$$


and

$$
\begin{aligned}
M_{2} & =\left(3 x^{4}+6 x^{2} y^{2}+3 y^{4}+8 x^{3}+12 x y^{2}+12 x^{2}\right. \\
& +12 x+12 t+300)^{2}
\end{aligned}
$$

At fixed time $\mathrm{t}$, one knows $U$ decays like $\frac{1}{r^{3}}$ for $r \rightarrow \infty$. Also, $U$ tends asymptotically to zero at the rate $\frac{1}{t}$ at any fixed point $(x, y)$ when $t \rightarrow \infty$.

- Example 2

Let $\mathcal{P}_{1}=\left(z^{3}+6 t\right)+2 i z$ and $\mathcal{P}_{2}=-i\left(z^{3}+6 t\right)+z$. Then a simple calculation can yield the imaginary part of $W$ in (7)

$f(x, y, t)=\left(x^{2}+y^{2}\right)^{3}+4 x^{3} y+8 x y^{3}+2\left(x^{2}+y^{2}\right)+6 t\left(2 x^{3}-6 x y^{2}-y\right)+36 t^{2}+6000$

From (28), we can see that

$$
f(x, y, 0) \approx\left(x^{2}+y^{2}\right)^{3} \quad \text { near } \quad r=\sqrt{x^{2}+y^{2}}=\infty .
$$

It can be verified that $f(x, y, 0)$ is positive for all $\mathbb{R}^{2}$. Also, letting (28) be equal to zero, one has

$$
\begin{aligned}
t & =(1 / 2) x y^{2}+(1 / 12) y-(1 / 6) x^{3} \\
& \pm(1 / 12) \sqrt{24 x^{2} y^{4}-20 x y^{3}-36 x^{4} y^{2}-7 y^{2}-20 x^{3} y-4 y^{6}-8 x^{2}-23996}
\end{aligned}
$$

A simple calculation shows that the equation inside the square root is negative for all $\mathbb{R}^{2}$. Hence $f(x, y, t)$ is positive for all $\mathbb{R}^{2}$ at any fixed time $t \geq 0$. Then the solution $U$ in (5) of the Novikov-Veselov equation (1) is

$$
U=\frac{F_{1}}{F_{2}},
$$

where

$$
\begin{aligned}
F_{1} & =-\frac{1}{2}\left[24 x^{7} y+16 x^{6}+24 x^{5} y^{3}+\left(432 t y+216000+48 y^{2}\right) x^{4}\right. \\
& +\left(-24 y^{5}-144 t+48 y\right) x^{3}+\left(432000 y^{2}+864 t y^{3}-96 y^{4}\right) x^{2} \\
& +\left(-24 y^{7}-48 y^{3}+432 t y^{2}+\left(432000+1728 t^{2}\right) y\right) x+48000 \\
& \left.+432 y^{5} t-32 y^{6}+216000 y^{4}+252 t^{2}\right]
\end{aligned}
$$

and

$$
\begin{aligned}
F_{2} & =\left(x^{6}+3 x^{4} y^{2}+3 x^{2} y^{4}+y^{6}+4 x^{3} y+8 x y^{3}+2 x^{2}+2 y^{2}\right. \\
& \left.+12 t x^{3}-36 t x y^{2}-6 t y+36 t^{2}+6000\right)^{2}
\end{aligned}
$$

At fixed time $\mathrm{t}$, one knows $U$ decays like $\frac{1}{r^{4}}$ for $r \rightarrow \infty$. Also, $U$ tends asymptotically to zero at the rate $\frac{1}{t^{2}}$ at any fixed point $(x, y)$ when $t \rightarrow \infty$. 
- Example 3

Let $\mathcal{P}_{1}=\left(z^{4}+24 t z\right)+2 i z$ and $\mathcal{P}_{2}=-i\left(z^{4}+24 t z\right)+z$. A tedious calculation shows that the imaginary part of $W$ in (17) is

$g(x, y, t)=\left(x^{2}+y^{2}\right)^{4}+6 x^{4} y+12 x^{2} y^{3}-(18 / 5) y^{5}+2 x^{2}+2 y^{2}+24 t\left(x^{2}+y^{2}\right)\left(2 x^{3}-6 x y^{2}+24 t\right)+100$

Similarly, $g(x, y, t)$ is positive for all $\mathbb{R}^{2}$ at fixed time $t \geq 0$. Then the solution $U$ in (5) can be written as

$$
U=\frac{G_{1}}{G_{2}}
$$

where

$$
\begin{aligned}
& G_{1}(x, y, t) \\
= & -\frac{1}{2}\left[240 y^{11}-1680 x^{2} y^{9}-1980 y^{8}+\left(34560 t x-5280 x^{4}\right) y^{7}\right. \\
+ & \left(160000-2160 x^{2}\right) y^{6}+\left(480+218880 t x^{3}-3360 x^{6}+138240 t^{2}\right) y^{5} \\
+ & \left(480000 x^{2}-9000 x^{4}\right) y^{4}+\left(-120000+1200 x^{8}+57600 t x^{5}\right. \\
+ & \left.\left(-1382400 t^{2}-4800\right) x^{2}\right) y^{3}+\left(3600 x^{6}-5760000 t x+480000 x^{4}\right) y^{2} \\
+ & \left(1200 x^{10}+57600 x^{7} t+\left(2400+691200 t^{2}\right) x^{4}+360000 x^{2}\right) y \\
+ & \left.20000+160000 x^{6}+1920000 t x^{3}+5760000 t^{2}+900 x^{8}\right]
\end{aligned}
$$

and

$$
\begin{aligned}
& G_{2}(x, y, t) \\
= & \left(5 x^{8}+20 x^{6} y^{2}+30 x^{4} y^{4}+20 x^{2} y^{6}+5 y^{8}+30 x^{4} y+60 x^{2} y^{3}-18 y^{5}+10 x^{2}\right. \\
+ & \left.10 y^{2}+240 t x^{5}-480 t x^{3} y^{2}+2880 x^{2} t^{2}-720 t y^{4} x+2880 y^{2} t^{2}+500\right)^{2}
\end{aligned}
$$

In this case, at fixed time, one knows that $U$ decays like $\frac{1}{r^{5}}$ for $r \rightarrow \infty$; moreover, $U$ also tends asymptotically to zero at the rate $\frac{1}{t^{2}}$ at fixed point $(x, y) \neq(0,0)$ when $t \rightarrow \infty$. We notice here that at $(0,0), U$ approaches $\infty$ as $t \rightarrow \infty$.

- Example 4

Let $\mathcal{P}_{1}=\left(z^{5}+60 t z^{2}\right)+2 i z$ and $\mathcal{P}_{2}=-i\left(z^{5}+60 t z^{2}\right)+z$. Then the imaginary part of $W$ in (7) is

$$
\begin{aligned}
h(x, y, t) & =\left(\left(x^{2}+y^{2}\right)^{5}-\frac{10}{3} x^{5} y+\frac{20}{3} x^{3} y^{3}+\left(x^{2}+y^{2}\right)\left(2+12 x^{3} y-12 x y^{3}\right)\right. \\
& +120 t\left(x^{2}+y^{2}\right)\left(x^{5}-3 y^{4} x-2 x^{3} y^{2}\right)+3600 t^{2}\left(x^{2}+y^{2}\right)^{2} \\
& \left.+20 t\left(11 y^{3}+3 x^{2} y\right)+120 t^{2}+1000\right)
\end{aligned}
$$


Similarly, $h(x, y, t)$ is positive for all $\mathbb{R}^{2}$ at fixed time $t \geq 0$. Then the solution $U$ in (5) is

$$
U=\frac{H_{1}}{H_{2}}
$$

where

$$
\begin{aligned}
& H_{1}(x, y, t) \\
= & -\frac{1}{2}\left[360 x y^{13}+\left(360 x^{3}+14400 t\right) y^{11}-144 y^{10}+\left(14400 x^{2} t-1800 x^{5}\right) y^{9}\right. \\
+ & \left(-720 x^{2}+900000+108000 t^{2}\right) y^{8}+\left(237600 t x^{4}-777600 t^{2} x-3600 x^{7}\right) y^{7} \\
+ & \left(3840 x^{4}+\left(3600000+432000 t^{2}\right) x^{2}+83520 t x\right) y^{6}+\left(165600 t x^{6}+720 x\right. \\
- & \left.10368000 t^{3}-1800 x^{9}-345600 t^{2} x^{3}\right) y^{5}+\left(-1120 x^{6}+(5400000\right. \\
+ & \left.\left.648000 t^{2}\right) x^{4}+12000 t x^{3}+\left(-129600000 t-15552000 t^{3}\right) x-810000 t^{2}\right) y^{4} \\
+ & \left(360 x^{11}-50400 x^{8} t+950400 x^{5} t^{2}-2160 x^{3}+20736000 t^{3} x^{2}+\left(-216000 t^{2}\right.\right. \\
- & 1800000) x-5760 t) y^{3}+\left(2380 x^{8}+\left(3600000+432000 t^{2}\right) x^{6}-25200 t x^{5}\right. \\
+ & \left.\left(-10368000 t^{3}-86400000 t\right) x^{3}+453600 x^{2} t^{2}+62208000 t^{4}+518400000 t^{2}\right) y^{2} \\
+ & \left(360 x^{13}+21600 x^{10} t+518400 x^{7} t^{2}+720 x^{5}+31104000 t^{3} x^{4}+(1920000\right. \\
+ & \left.\left.230400 t^{2}\right) x^{3}+17280 x^{2} t+1555200 t^{3}+12960000 t\right) y+476 x^{10} \\
+ & \left(900000+108000 t^{2}\right) x^{8}+25200 x^{7} t+\left(43200000 t+5184000 t^{3}\right) x^{5} \\
+ & \left.\left.226800 x^{4} t^{2}+\left(62208000 t^{4}+518400000 t^{2}\right) x^{2}+72000+8640 t^{2}\right)\right]
\end{aligned}
$$

and

$$
\begin{aligned}
& H_{2}(x, y, t) \\
= & \left(3 x^{10}+15 x^{8} y^{2}+30 x^{6} y^{4}+30 y^{6} x^{4}+15 y^{8} x^{2}+3 y^{10}+26 x^{5} y+20 x^{3} y^{3}\right. \\
+ & 6 x^{2}+6 y^{2}-36 x y^{5}+360 x^{7} t-1800 t x^{3} y^{4}-360 t x^{5} y^{2}-1080 t x y^{6} \\
+ & \left.10800 x^{4} t^{2}+21600 t^{2} x^{2} y^{2}+10800 t^{2} y^{4}+660 t y^{3}+180 t x^{2} y+360 t^{2}+3000\right)^{2}
\end{aligned}
$$

In this case, at fixed time, $U$ decays like $\frac{1}{r^{6}}$ for $r \rightarrow \infty$; moreover, we see that

$$
U \rightarrow \frac{-240\left(x^{2}+y^{2}\right)}{\left[30\left(x^{2}+y^{2}\right)^{2}+1\right]^{2}}=\frac{-240 z \bar{z}}{\left[30 z^{2} \bar{z}^{2}+1\right]^{2}} \quad \text { as } \quad t \rightarrow \infty
$$

at fixed point $(x, y)$, which is a stationary solution of (1) for

$$
V=\frac{3600 \bar{z}^{4} z^{2}-120 \bar{z}^{2}}{\left(30 z^{2} \bar{z}^{2}+1\right)^{2}} .
$$

We notice that if we define

$$
u(z, \bar{z})=\ln U \quad \text { and } \quad V(z, \bar{z})=\frac{U_{z z}}{3 U},
$$


then the stationary equation of the Novikov-Veselov equation (1) will become the Tzitzeica equation [9, 16]

$$
u_{z \bar{z}}=e^{u}+\epsilon e^{-2 u},
$$

where $\epsilon$ is an arbitrary constant. It can be verified that (29) satisfies the $\epsilon=0$ case, i.e., the Liouville equation, whose real solutions are given by

$$
\ln \frac{-2 \kappa\left|\frac{d S}{d z}\right|^{2}}{\left(1+\kappa|S|^{2}\right)^{2}}
$$

where $S(z)$ is a locally univalent meromorphic function in some domain and $\kappa$ is a constant. For the solution corresponding to (29), one knows that $S(z)=z^{2}$ and $\kappa=30$.

For general case, let's choose

$$
\mathcal{P}_{1}=P_{N}(t, z)+2 i z \quad \text { and } \quad \mathcal{P}_{2}=-i P_{N}(t, z)+z .
$$

We can expect the imaginary part of $W$ in (7) is positive for all $\mathbb{R}^{2}$ at any fixed time if we choose an appropriate constant. And the solution $U(x, y, t)$ at any fixed time decays like $\frac{1}{r^{N+1}}$ for $N \geq 3$. For $N=1$, one refers to [13]. It could be interesting to study the asymptotic solutions when $t \rightarrow \infty$ and then one obtains the solutions (31) of the Liouville equation or the ones of the Tzitzeica equation (30). One remarks here that in these examples, for each potential $U(x, y, t)$ there exist infinitely many wave functions, which can be constructed by the Pfaffian [1, 25] of linear combinations of the Gould-Hopper polynomials. Hence it could be worthwhile to investigate the corresponding wave functions when $t \rightarrow \infty$. But the computations are more involved and the details will be published elsewhere.

\section{Concluding Remarks}

In this paper we have studied the Novikov-Veselov equation using the Gould-Hopper polynomials. Firstly, one investigates the root dynamics of the so-called $\sigma$-flows and gets the Lax pair; moreover, one finds that when $N=3,4,5$, the root dynamics satisfies the Gold-Fish model. Although the Lax pair is established, only two conserved densities are found. The reason is that the Lax operator is nilpotent. Also, the asymptotic behavior is studied. Secondly, we construct smooth rational solutions using the Gould-Hopper polynomials and the skew product (7); besides, the asymptotic behaviors of the smooth rational solutions are discussed.

\section{Acknowledgments}

The author is grateful to Prof. Iskander Taimanov and Prof. Sergy Tsarev for valuable discussions when they visited Taiwan. He also thanks Prof. Wen-Xiu Ma 
for his suggestions. This work is supported in part by the National Science Council of Taiwan under Grant No. NSC 98-2115-M-606-001-MY2. 


\section{Appendix}

\section{A Gold-Fish Model}

For the Goldfish Model

$$
\ddot{q}_{i}=2 \sum_{j \neq i} \frac{\dot{q}_{i} \dot{q}_{j}}{\dot{q}_{i}-q_{j}},
$$

its initial value problem can be solved by the statement: $z=q_{i}(t), i=1,2, \cdots, N$ are the $\mathrm{N}$ roots of the equation [5]

$$
\sum_{i=1}^{N} \frac{\dot{q}_{i}(0)}{z-q_{i}(0)}=\frac{1}{t}
$$

It can be seen that it is a polynomial in $z$ with coefficients linear in $t$. Then the special choices of initial datum can get the solutions of the root dynamics (16) for the cases $N=3,4,5$. To illustrate it, we take $N=3$ as an example. When $N=3$, we have

$$
\frac{\dot{q}_{1}(0)}{z-q_{1}(0)}+\frac{\dot{q}_{2}(0)}{z-q_{2}(0)}+\frac{\dot{q}_{3}(0)}{z-q_{3}(0)}=\frac{1}{t}
$$

After some calculations, one yields

$$
\begin{aligned}
& z^{3}-z^{2}\left[\left(q_{1}(0)+q_{2}(0)+q_{3}(0)\right]+z\left[q_{1}(0) q_{2}(0)+q_{2}(0) q_{3}(0)+q_{1}(0) q_{3}(0)\right]-q_{1}(0) q_{2}(0) q_{3}(0)\right. \\
& =t z^{2}\left[\dot{q}_{1}(0)+\dot{q}_{2}(0)+\dot{q}_{3}(0)\right]-t z\left[\dot{q}_{1}(0)\left(q_{2}(0)+q_{3}(0)\right)+\dot{q}_{2}(0)\left(q_{1}(0)+q_{3}(0)\right)\right. \\
& \left.+\dot{q}_{3}(0)\left(q_{1}(0)+q_{2}(0)\right)\right]+t\left[\dot{q}_{1}(0) q_{2}(0) q_{3}(0)+\dot{q}_{2}(0) q_{1}(0) q_{3}(0)+\dot{q}_{3}(0) q_{1}(0) q_{2}(0)\right] .
\end{aligned}
$$

On the other hand, from (17), one knows $q_{1}(t), q_{2}(t), q_{3}(t)$ are the roots of the polynomial

$$
\mathbb{P}_{3}(z, t)=z^{3}+6 t+C_{1} z^{2}+C_{2} z+C_{3}
$$

or

$$
z^{3}+C_{1} z^{2}+C_{2} z+C_{3}=-6 t .
$$

Comparing (A.1) with (A.2), we are able to get the following linear equations for $\dot{q}_{1}(0), \dot{q}_{2}(0), \dot{q}_{3}(0)$ :

$$
\begin{aligned}
& \dot{q}_{1}(0)+\dot{q}_{2}(0)+\dot{q}_{3}(0)=0 \\
& \dot{q}_{1}(0)\left(q_{2}(0)+q_{3}(0)\right)+\dot{q}_{2}(0)\left(q_{1}(0)+q_{3}(0)\right)+\dot{q}_{3}(0)\left(q_{1}(0)+q_{2}(0)\right)=0 \\
& \dot{q}_{1}(0) q_{2}(0) q_{3}(0)+\dot{q}_{2}(0) q_{1}(0) q_{3}(0)+\dot{q}_{3}(0) q_{1}(0) q_{2}(0)=-6 .
\end{aligned}
$$

So if the determinant of the matrix

$$
\left(\begin{array}{ccc}
1 & 1 & 1 \\
q_{2}(0)+q_{3}(0) & q_{1}(0)+q_{3}(0) & q_{1}(0)+q_{2}(0) \\
q_{2}(0) q_{3}(0) & q_{1}(0) q_{3}(0) & q_{1}(0) q_{2}(0)
\end{array}\right)
$$


is not equal to zero, then the initial velocities $\dot{q}_{1}(0), \dot{q}_{2}(0), \dot{q}_{3}(0)$ can be uniquely expressed by the initial positions $q_{1}(0), q_{2}(0), q_{3}(0)$. For the cases $N=4,5$, the linear equations (A.3) and the matrix (A.4) can be obtained similarly.

\section{References}

[1] C.Athorne and J.J.C.Nimmo: On the Moutard transformation for integrable partial differential equations. Inverse problems 7 (1991), 809-826.

[2] M.Yu. Basalaev, V.G. Dubrovsky and A.V. Topovsky, New exact multi line soliton and periodic solutions with constant asymptotic values at infinity of the NVN integrable nonlinear evolution equation via dibar-dressing method, arXiv: 0912.2155, 2009, 43 pages,

[3] L.V. Bagdanov, The Veselov-Novikov equation as a natural two-dimensional Generalization of the KdV equation, Ther. Math. Fiz, 70, 309(1987)

[4] Gabriella Bretti and Paolo E. Ricci, Multiextensions of the Bernoulli and Appell polynomials, Taiwan Jour. of Math., Vol. 8, No. 3 (2004), 415-428,

[5] F. Calogero, The neatest many-body problem amenable to exact treatments (a "goldfish"?), Physica D, 152-153 (2001), pp. 78-84

[6] B.A. Dubrovin, I.M. Krichever,, and S.P. Novikov,: The Schordinger equation in a periodic field and Riemann surfaces. Soviet Math. Dokl. 17 (1976), 947-952.

[7] V.G. Dubrovsky and I.B. Formusatik, The construction of exact rational solutions with constant asymptotic values at infinity of two-dimensional NVN integrable nonlinear evolution equations via dbar-dressing method, J.Phys.A.: Math. and Gen., v.34A, 1837-1851 (2001).

[8] V.G. Dubrovsky and I.B. Formusatik, New lumps of Veselov-Novikov equation and new exact rational potentials of two-dimensional Schrodinger equation via dbar-dressing method, Phys. Lett., 2003, V.313, 1-2, 68-76.

[9] Ferapontov E.V., Stationary Veselov-Novikov equation and isothermally asymptotic surfaces in projective differential geometry, Preprint SFB 288 No. 318, Berlin (1998), arXiv:DG/9805001

[10] H.W. Gould and A.T. Hopper, Operational formulas connected with two generalizations of Hermite polynomials, Duke Math. J. , 29(1962), 51-63

[11] Oktay K. Pashaev and Zeynep Nilhan Gurkan, Abelian Chern-Simons Vortices and Holomorphic Burgers' Hierarchy, Theor.Math.Phys., 152(2007), 1017-1029 
[12] P.G. Grinevich, and S.V. Manakov,: Inverse scattering problem for the twodimensional Schrodinger operator, the dbar-method and nonlinear equations. Funct. Anal. Appl. 20:2 (1986), 94-103.

[13] P.G. Grinevich, Rational solitons of the Veselov-Novikov equation - reflectionless at fixed energy two-dimensional potentials, Teor. Mat. Fiz., Vol. 69, 307 (1986). 1170-1172

[14] P. Grinevich , A. Mironov and S. Novikov, New Reductions and Nonlinear Systems for 2D Schrodinger Operators, arXiv:1001.4300, 2010

[15] A.E.Mironov, The Novikov-Veselov hierarchy of equations and integrable deformations of minimal Lagrangian tori in $C P^{2}$, Siberian Electronic Mathematical Reports, V.1(2004), pp. 38-46

[16] A.E.Mironov, Relationship Between Symmetries of the Tzitzeica Equation and the Novikov-Veselov Hierarchy, Mathematical Notes, 2007, Vol. 82, No.4, pp. 569-572

[17] Heng-Chun Hu, Sen-Yue Lou, and Qing-Ping Liu: Darboux transformation and variable separation approach: the Nizhnik-Novikov-Veselov equation. Chinese Phys. Lett. 20 (2003), 1413-1415.

[18] Heng-Chun Hu and Sen-Yue Lou,Construction of the Darboux Transformaiton and Solutions to the Modified Nizhnik-Novikov-Veselov Equation, Chinese Phys. Lett. 21 , No.11 (2004), 2073-2076

[19] I.Krichever, A characterization of Prym varieties, Int. Math. Res. Not. 2006, Art. ID 81476, 36 pp.

[20] B.G. Konopelchenko, Introduction to multidimensional integrable equations: the inverse spectral transform in 2+1-dimensions, New York - London, Plenum Press, 1992.

[21] B. G. Konopelchenko and G. Landolfi, Induced Surfaces and Their Integrable Dynamics II: Generalized Weierstrass Representations in 4-d Spaces and Deformations via DS Hierarchy, Studies in Applied Mathematics, Vol.104(2000), 129-169

[22] Wen-Xiu Ma and Yuncheng You, Solving the Korteweg-de Vries Equation by Its Bilinear Form: Wronskian Solutions, Trans. Amer. Math. Soc., 357 (2005), No.5, 1753-1778

[23] S.V. Manakov, USP. Matem. Nauk, Vol.31, No.5, p.245, 1976 
[24] C. R. Moutard, Acad. Sci. Poris 80 729, 1875 ; C. R. Moutard, J. de L'Ecole Polpehnique Cahier 45 I, 1878

[25] Nimmo J.J.C. Darboux transformations in $(2+1)$ dimensions, in: Proc. NATO ARW Applications of Analytic and Geometric Methods to Nonlinear Differential Equations (ed. P. Clarkson), NATO ASI Series, Kluwer, 1992, p. 183V192.

[26] S.P. Novikov, Two-dimensional Schrodinger operators in periodic fields, J. Sov. Math., 28(1), 1-20,1985

[27] S.P. Novikov and A.P. Veselov, Two-dimensional Schordinger operator: Inverse scattering transform and evolutional equations, Physica D, 18 (1-3), 267-273 (1986).

[28] Si-Qi Liu, Chao-Zhong Wu and Youjin Zhang, On the Drinfeld-Sokolov Hierarchies of D type, arXiv:0912.5273, 2009

[29] V.B. Matveev, M.A. Salle, Darboux Transformations and solitons, in Springer series in Nonlinear Dynamics, Springer, Berlin, Heidelberg, 1991.

[30] Y.Ohta, Pfaffian solution for the Veselov-Novikov equation, Jour. of the Phys. Soc. of Japan, Vol. 61, No. 11(1992), 3928-3933

[31] T.Shiota, Prym varieties and soliton equations. Infinite-dimensional Lie algebras and groups (Luminy-Marseille, 1988), 407-448, Adv. Ser. Math. Phys., 7, World Sci. Publ., Teaneck, NJ, 1989.

[32] I.A. Taimanov and S.P. Tsarev, Two-dimensional rational solitons and their blow-up via the Moutard transformation, Theoret. and Math. Phys. 157, No.2, (2008)

[33] Kanehisa Takasaki, Dispersionless Hirota equations of two-component BKP hierarchy, SIGMA, Vol. 2(2006), Paper 057, 22 pages, arXiv:nlin.SI/0604003

[34] A.P. Veselov and S.P. Novikov, Finite-zone, two-dimensional, potential Schordinger operators. Explicit formulas and evolution equations, Sov. Math., Dokl. 30(1984), 588-591 